\title{
Dialogfähiges programmierbares Infusionssystem
}

\author{
J. Nagel, H. Müller, W. Schubert, R. Thull, M. Schaldach \\ Zentralinstitut für Biomedizinische Technik, Universität Erlangen-Nürnberg
}

Einige wichtige Medikamente können nicht oral verabreicht werden, weil sie aufgrund ihrer chemischen Struktur im Magen-Darm-Trakt entweder nicht absorbiert oder so verändert werden, daß sie ihre Wirksamkeit verlieren. Andere Medikamente missen so exakt dosiert werden, daß eine orale Gabe wegen der aus den Halbwertszeiten resultierenden Konzentrationsschwankungen nicht in Frage kommt.

Diese Probleme können nur mit Hilfe eines Infusionssystems gelöst werden, das in der Lage ist, den jeweiligen Medikamentenbedarf direkt in das Blut oder das Körpergewebe abzugeben. Mit Hilfe der kontinuierlichen gesteuerten oder geregelten Infusion läBt sich eine optimale Medikamentenkonzentration aufrecht erhalten. Zur Abgabe kleinster Medikamentenmengen wurde eine extrakorporale, tragbare Infusionseinheit entwickelt, die zunächst für die programierte Insul indosierung bei der Diabetes-Therapie zur Anwendung kormt.

Das künstliche Pankreas gilt bei Patienten mit schwerem Diabetes mellitus als Therapie der Wahl. In einigen Laboratorien sind unterschiedliche extrakorporale Geräte entwickelt worden, die als geregelte Systeme auf der Basis einer quasi-kontinuierlichen Insulin-Abgabe arbeiten und über einen Glukose-Sensor als Meßwertgeber verfügen. Trotz zahlreicher Versuche ist es bisher jedoch nicht gelungen, einen Glukose-Sensor zu entwickeln, der für einen Langzeiteinsatz in einem tragbaren extrakorporalen oder in einem implantierbaren System geeignet ist. Aus diesem Grund können derzeit tragbare Geräte ausschlieBlich als "open-10op" Systeme ohne Glukose-Sensor realisiert werden. Hierbei erfolgt die Insulin-Abgabe in Anlehung an vorprogrammierte Werte, die für den Patienten mit Hilfe eines stationären sensor-geregelten Systems ermittelt werden.

Das beschriebene Gerät besteht aus drei Funktionsblöcken, nämlich der Insulin-Pumpe, dem Pumpenantrieb und einer elektronischen Steuereinheit, die die jeweilige fördermenge vorgibt (Abb. 1). Als Pumpe wird eine Hamilton-Spritze benutzt mit einem Volumen von wahlweise 2,5 oder $5 \mathrm{ml}$. Der Antrieb erfolgt über einen Spindel-Trieb, der von einem Elektromotor angetrieben wird. Motor und Elektronik des Gerätes sind batteriegetrieben.

Die mit einem Mikroprozessor realisierte Steuerelektronik hat die Aufgabe, aus den jeweils zu fordernden momentanen Dosierraten den erforderlichen Vorschub der Infusionsspritze zu berechnen, den Antriebsmotor entsprechend zu steuern und die Insul införderung zu überwachen. Die patientenspezifischen Infusionsprofile stehen wahlweise in einem programierbaren Festwertspeicher (EPROM), der in die Steuerelektronik integriert ist, zur Verfügung, oder werden uber eine serielle Schnittstelle von einem externen Datengeber (Glukoseregler) abgerufen. Neben den Infusionsraten können alle weiteren Betriebsparameter über die Schnittstelle abgefragt, bzw. umprogramiert werden.

Bei Benutzung des internen Datenspeichers stehen 8 verschiedene Infusionsprofile zu Verfügung, die vom Patienten uber eine Tastatur abgerufen werden können. Daneben wird automatisch, kontinuierlich 
ein Basisprofil gefördert, dessen Werte durch Eingabe eines Faktors uber die Tastatur angehoben oder abgesenkt werden können. Ober eine LCD-Anzeige, die zur Funktionskontrolle der Dosiereinheit dient, kann der jeweilige Status des Gerätes abgefragt werden.

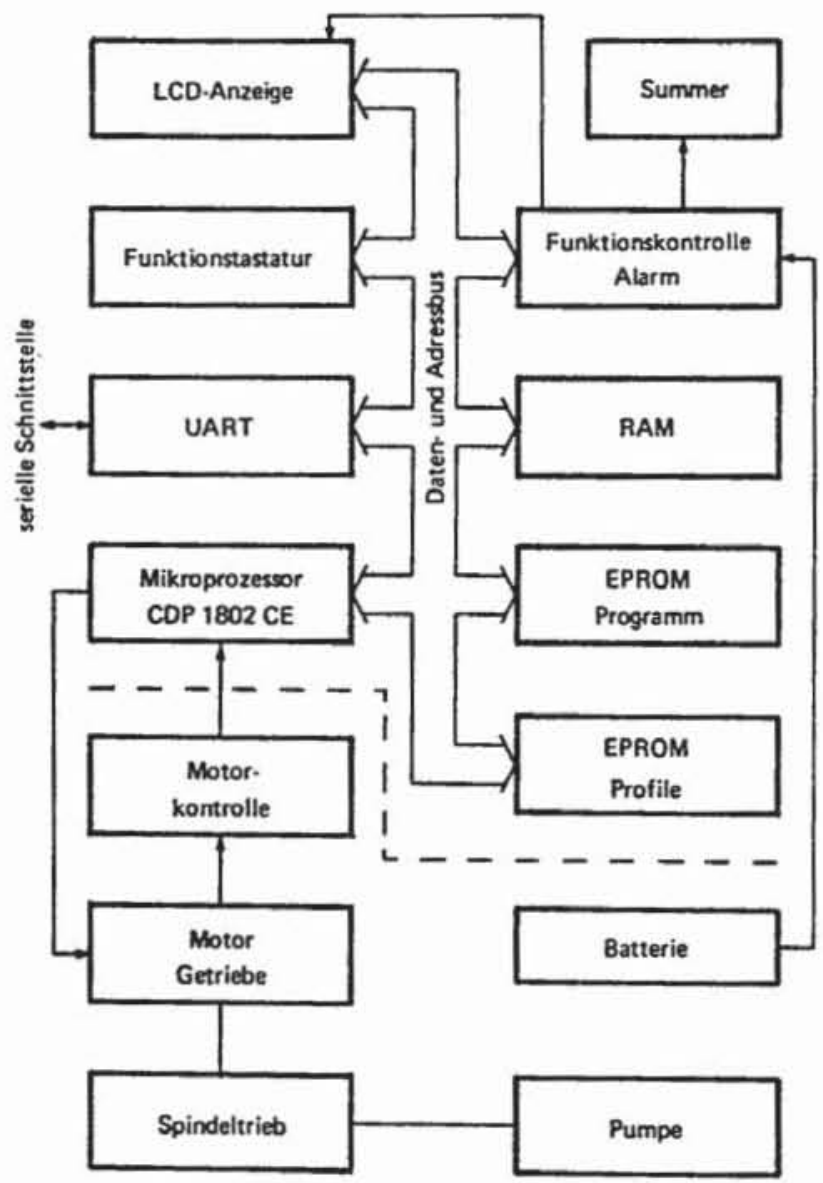

Abb. 1 Blockschaltbild des Infusionssystems

Bei Störungen in der Dosiereinheit erfolgt ein optischer und akustischer Alarm. Zur Erhöhung der Patientensicherheit beim Betrieb der Dosiereinheit mit dem separaten Glukoseregler wurde eine Notfallsteuerung implementiert. Treffen über einen längeren Zeitraum vom Glukoseregler keíne Signale mehr ein, oder sind diese gestört, so schaltet die Dosiereinheit auf Konstantförderung um, d.h. es wird pro Zeiteinheit eine konstante Menge ausgeschuttet, und zwar der zuletzt vom Glukoseregler empfangene Basalwert, wodurch eine Hyperglykämie verhindert wird.

Zur Programierung des Gerätes müssen die mittels stationärem System ermittelten patientenspezifischen Infusionsprofile in den Festwertspeicher eingegeben werden. Zur Erleichterung der Datenübertragung wurde ein interaktives graphisches Program-

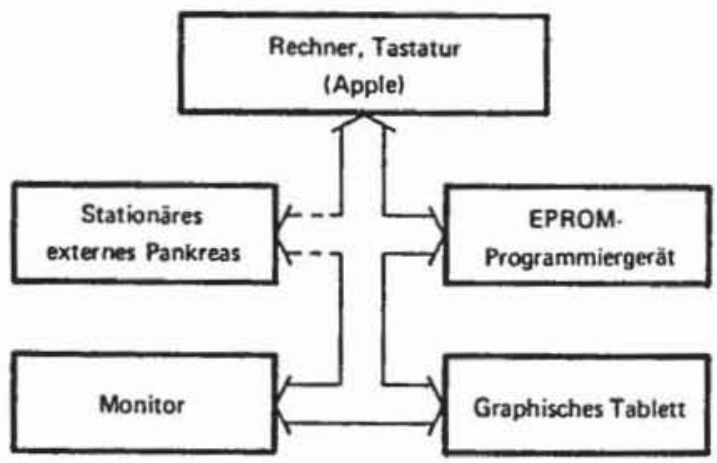

Abb. 2 Programiersystem

miersystem aufgebaut (Abb. 2). Das System ermöglicht die wahlweise Eingabe der Profile in den Festwertspeicher uber eine direkte Kopplung von stationärem Pankreas und Programmiersystem, über eine TeletypeTastatur oder durch Abtastung der aufgezeichneten Profilf uber ein "graphisches Tablett". Auf einem Monitor werden die Profile graphisch oder alphanumerisch dargestellt, so daß sie einfach geprift und verändert, bzw. korrigiert werden können. Die eingegebenen Daten werden in einem EPROM gespeichert, das dann als Datenträger in das extrakorporale Infusionssystem eingesetzt wird.

Zur weiteren Verbesserung der Einsatzmöglichkeiten erfolgt die Miniaturisierung des Systems. Durch den Einsatz einer energiesparenden Pumpe und eines Telemetrie-Systems soll weiterhin die Möglichkeit einer Implantation des Infusionssystems geschaffen werden. 


\section{Literatur:}

1. Albisser, A.M., Jackman, W.S., Ferguson, R., Bahoric, A. and Goriya, Y. (1978). A portable precision pumping system for chronic, programmed insul in infusion. Med. Progr. Technol. 5, 187-193

2. Blackshear, P.J., Rohde, T.D., Prosl, F. and Buchwald, H. (1979). The implantable infusion pump: a new concept in drug delivery. Med. Progr. Technol. 6, 149-161
3. Summers, G.B. (1970). A new and growing family of artificial implanted fluid devices. Trans. Amer. Soc. Artif. Int. Organs 16, 218

4. Zaffaroni, A. (1976). Drug delivery systems. MEDEX 76, Base1 